\title{
Towards Transversality of Singular Varieties: Splayed Divisors
}

\author{
by \\ Eleonore FABER
}

\begin{abstract}
We study a natural generalization of transversally intersecting smooth hypersurfaces in a complex manifold: hypersurfaces whose components intersect in a transversal way but may be themselves singular. Such hypersurfaces will be called splayed ${ }^{1}$ divisors. A splayed divisor is characterized by a property of its Jacobian ideal. This yields an effective test for splayedness. Two further characterizations of a splayed divisor are shown, one reflecting the geometry of the intersection of its components, the other one using K. Saito's logarithmic derivations. As an application we prove that a union of smooth hypersurfaces has normal crossings if and only if it is a free divisor and has a radical Jacobian ideal. Further it is shown that the Hilbert-Samuel polynomials of splayed divisors have the natural additivity property.
\end{abstract}

2010 Mathematics Subject Classification: Primary 32S25; Secondary 32S10, 13D40.

Keywords: normal crossing divisor, free divisor, logarithmic derivations, Jacobian ideal, Hilbert-Samuel polynomial.

\section{$\S 1$. Introduction}

Let $M_{1}, M_{2}$ be two submanifolds of a complex manifold $S$. Then $M_{1}$ and $M_{2}$ intersect transversally at a point $p \in S$ if their respective tangent spaces add up to the tangent space of $S$ at $p$, that is,

$$
T_{p} M_{1}+T_{p} M_{2}=T_{p} S .
$$

Transversality is a fundamental concept in algebraic geometry as well as in differential geometry. However, in many applications (e.g., embedded resolution of

Communicated by S. Mukai. Received July 10, 2012. Revised September 9, 2012.

E. Faber: Department of Computer and Mathematical Sciences,

University of Toronto at Scarborough, Toronto, Ont. M1A 1C4, Canada. and

Fakultät für Mathematik, Universität Wien, Austria;

e-mail: efaber@math.toronto.edu, eleonore.faber@univie.ac.at

1 The term "splayed" means "spread out" or "made oblique". So being "splayed" for a hypersurface $D=D_{1} \cup D_{2}$ should indicate that the components $D_{1}$ and $D_{2}$ are "spread out" in the ambient space.

(C) 2013 Research Institute for Mathematical Sciences, Kyoto University. All rights reserved. 
singularities, Hodge structures) one needs a notion of transversality for more than two subspaces. This leads to the notion of normal crossings, which means that several smooth components cross in a transversal way. Another way to phrase this is that the union of several smooth subspaces is isomorphic to a union of coordinate subspaces.

In this article we study a natural generalization of the concept of transversal intersection of two hypersurfaces in complex manifolds allowing singular components. The geometric idea is that two singular hypersurfaces $D_{1}$ and $D_{2}$ in a complex manifold $S$ intersect transversally at a point $p$ if their "tangent spaces" fill out the whole space and the ideal of their intersection is reduced. The notion of tangent space for singular hypersurfaces can be made precise by means of logarithmic derivations. In algebraic terms this means: one can find local coordinates at $p$ such that the defining equations of the $D_{i}$ can be chosen in separated variables. We call a union of such transversally intersecting hypersurfaces a splayed divisor. This concept has already appeared in different contexts under different names, for example, J. Damon [Dam96] called germs of the form $\left(V_{1} \times \mathbb{C}^{m}\right) \cup\left(\mathbb{C}^{n} \times V_{2}\right) \subseteq \mathbb{C}^{n+m}$, where $V_{1} \subseteq \mathbb{C}^{n}$ and $V_{2} \subseteq \mathbb{C}^{m}$, a product union (see also Remark 2.5 below). For an example of a splayed divisor in a three-dimensional $S$, see Figure 1.
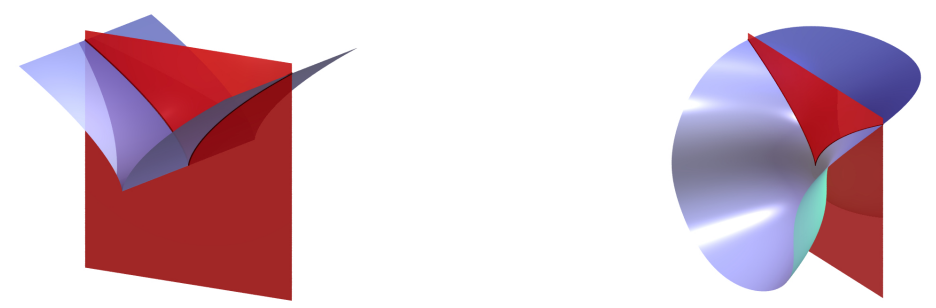

Figure 1. $D=\left\{x\left(y^{2}-z^{3}\right)=0\right\}$ (left) is splayed and $D^{\prime}=\left\{x\left(x+y^{2}-z^{3}\right)=0\right\}$ (right) is not splayed.

In the first part of this work three different characterizations of splayed divisors are shown. Suppose that a divisor $D \subseteq S$ is given by $(D, p)=\left(D_{1}, p\right) \cup$ $\left(D_{2}, p\right)=\{g h=0\}$. First we consider the Jacobian ideal $J_{g h}$ (the ideal generated by the partial derivatives of $g h$ ) of $D$ at $p$. It is clear that for a splayed $D$, the Jacobian ideal satisfies

$$
J_{g h}=g J_{h}+h J_{g}
$$

when the defining equations $g$ and $h$ are chosen in separated variables. We show (Proposition 3.3) that this property already characterizes splayed divisors. 
Further, for a splayed divisor $D$, the ideal $\left((g h)+J_{g h}\right)$ defining its singular locus can be written as the intersection of the ideals defining the singular loci of the $D_{i}$ and of the ideal defining the intersection $D_{1} \cap D_{2}$ :

$$
\left((g h)+J_{g h}\right)=(g, h) \cap\left((g)+J_{g}\right) \cap\left((h)+J_{h}\right) .
$$

In Proposition 3.5 the sufficiency of this condition is proved.

Finally, equation (1.1) can be directly translated to singular subspaces via K. Saito's logarithmic derivations [Sai80], namely, $D$ is splayed at $p$ if and only if

$$
\operatorname{Der}_{S, p}\left(\log D_{1}\right)+\operatorname{Der}_{S, p}\left(\log D_{2}\right)=\operatorname{Der}_{S, p} .
$$

This is shown in Proposition 3.10.

In the second part, two applications are considered. First the relationship between splayed divisors and free divisors is studied. Free divisors are a generalization of normal crossing divisors and appear frequently in different areas of mathematics, for example, in deformation theory as discriminants or in combinatorics as free hyperplane arrangements; see [Ale86, OT92, BEvB09, MS10, BM06, Sai81] for more examples. Then we give a partial answer to a question of $\mathrm{H}$. Hauser about the characterization of normal crossing divisors by their Jacobian ideals: it is shown that if $D=\bigcup_{i=1}^{n} D_{i}$ is locally the union of smooth irreducible components then $D$ has normal crossings if and only if $D$ is locally free and its Jacobian ideal is radical.

The computation of singularity invariants of splayed divisors is very interesting, in particular one should be able to deduce them from singularity invariants of their splayed components. We start by computing the Hilbert-Samuel polynomial $\chi_{D, p}$ of a splayed divisor $(D, p)=\left(D_{1}, p\right) \cup\left(D_{2}, p\right)$ and find that it satisfies an additivity relation: from the exact sequence

$$
0 \rightarrow \mathcal{O}_{D, p} \rightarrow \mathcal{O}_{D_{1}, p} \oplus \mathcal{O}_{D_{2}, p} \rightarrow \mathcal{O}_{D_{1} \cap D_{2}, p} \rightarrow 0
$$

we deduce

$$
\chi_{D, p}(t)+\chi_{D_{1} \cap D_{2}, p}(t)=\chi_{D_{1}, p}(t)+\chi_{D_{2}, p}(t) .
$$

This additivity relation does not characterize splayed divisors, an example therefore is given at the end.

\section{$\S 2$. Setting and triviality lemma}

We work in the complex analytic category. The main objects of our study are divisors (= hypersurfaces) in complex manifolds. We write $(S, D)$ for a fixed divisor $D$ in an $n$-dimensional complex manifold $S$. Denote by $p$ a point in $S$ and 
by $x=\left(x_{1}, \ldots, x_{n}\right)$ the complex coordinates of $S$ around $p$. The divisor $(D, p)$ will then be defined locally by an equation $h_{p}\left(x_{1}, \ldots, x_{n}\right)=0$ where $h_{p} \in \mathcal{O}_{S, p} \cong$ $\mathbb{C}\left\{x_{1}, \ldots, x_{n}\right\}$ (if the context is clear we omit the subscript ${ }_{p}$ ). Note that we will always assume that $h$ is reduced! To simplify the notation regarding splayed divisors, sometimes the coordinates are denoted by $\left(x_{1}, \ldots, x_{k}, y_{k+1}, \ldots, y_{n}\right)$ and $h(x, y) \in \mathbb{C}\{x, y\}$. The divisor $D$ has normal crossings at a point $p$ if one can find complex coordinates $\left(x_{1}, \ldots, x_{n}\right)$ at $p$ such that the defining equation $h$ of $D$ is $h=x_{1} \cdots x_{k}$ for $k \leq n$. We also say that $(D, p)$ is a normal crossing divisor. The Jacobian ideal of $h$ is denoted by $J_{h}=\left(\partial_{x_{1}} h, \ldots, \partial_{x_{n}} h\right) \subseteq \mathcal{O}_{S, p}$. The image of $J_{h}$ under the canonical epimorphism that sends $\mathcal{O}_{S, p}$ to $\mathcal{O}_{D, p}=\mathcal{O}_{S, p} /(h)$ is denoted by $\widetilde{J_{h}}$. The associated analytic coherent ideal sheaves are denoted by $J \subseteq \mathcal{O}_{S}$ and $\widetilde{J}$ in $\mathcal{O}_{D}$. The singular locus Sing $D$ of $D$ is defined by the ideal sheaf $\widetilde{J} \subseteq \mathcal{O}_{D}$. The singular locus (Sing $D, p$ ) is defined by the local $\operatorname{ring} \mathcal{O}_{\operatorname{Sing} D, p}=\mathcal{O}_{S, p} /\left((h)+J_{h}\right)$.

The next lemma yields an ideal-theoretic characterization of Cartesian product structure. It is used frequently and can be found in various different formulations in the literature (for example in [dJP00, Sai80, GH85, CNM96]).

Lemma 2.1 (Triviality lemma). Let $(S, p)$ locally be isomorphic to $\left(\mathbb{C}^{n+m}, 0\right)$ and denote $\mathcal{O}_{S, p}=\mathbb{C}\left\{x_{1}, \ldots, x_{n}, y_{1}, \ldots, y_{m}\right\}$ and let $h\left(x_{1}, \ldots, x_{n}, y_{1}, \ldots, y_{m}\right)$ be an element of $\mathcal{O}_{S, p}$. Then the following are equivalent:

(a) The ideal $\left(\partial_{y_{1}} h, \ldots, \partial_{y_{m}} h\right)$ is contained in the ideal $\left(h, \partial_{x_{1}} h, \ldots, \partial_{x_{n}} h\right)$.

(b) There exists a local biholomorphic map $\varphi:\left(\mathbb{C}^{n+m}, 0\right) \rightarrow\left(\mathbb{C}^{n+m}, 0\right)$ and a holomorphic $v(x, y) \in \mathcal{O}_{S, p}^{*}$ such that

$$
\begin{array}{r}
\varphi(x, y)=\left(\varphi_{1}(x, y), \ldots, \varphi_{n}(x, y), y_{1}, \ldots, y_{m}\right), \\
\varphi(x, 0)=(x, 0), v(x, 0) \equiv 1 \text { and } h \circ \varphi(x, y)=v(x, y) h(x, 0)
\end{array}
$$

This means that $D=\{h(x, y)=0\}$ is locally at $p$ isomorphic to some $\left(D^{\prime} \times \mathbb{C}^{m},(0,0)\right)$ where $D^{\prime}$ is locally contained in $\mathbb{C}^{n}$.

Remark 2.2. This lemma is called the "triviality lemma" because a stronger form of it characterizes local analytic triviality: if in (a) all partial derivatives $\partial_{y_{i}} h$ are even contained in $\left(x_{1}, \ldots, x_{n}, y_{1}, \ldots, y_{m}\right)\left(\partial_{x_{1}} h, \ldots, \partial_{x_{n}} h\right)$, then one can show that $(D, p) \cong\left(D_{0} \times \mathbb{C}^{m},\left(p^{\prime}, 0\right)\right)$ where $D_{0}=\{h(x, 0)=0\}$ is the "fibre" at the origin.

Proof. See for example [Sai80].

The objects of our studies are divisors that are unions of Cartesian products. We call them splayed because they fill out the whole space. 
Definition 2.3. Let $D$ be a divisor in a complex manifold $S$ with $\operatorname{dim} S=n$. The divisor $D$ is called splayed at a point $p \in S$ (or $(D, p)$ is splayed) if one can find coordinates $\left(x_{1}, \ldots, x_{n}\right)$ at $p$ such that $(D, p)=\left(D_{1}, p\right) \cup\left(D_{2}, p\right)$ is defined by

$$
h(x)=h_{1}\left(x_{1}, \ldots, x_{k}\right) h_{2}\left(x_{k+1}, \ldots, x_{n}\right),
$$

for some $k$ with $1 \leq k \leq n-1$, where $h_{i}$ is the defining reduced equation of $D_{i}$. Note that the $h_{i}$ are not necessarily irreducible. The splayed components $D_{1}$ and $D_{2}$ are not unique. Splayed means that $D$ is the union of two products: since $h_{1}$ is independent of $x_{k+1}, \ldots, x_{n}$, the divisor $D_{1}$ is locally at $p$ isomorphic to a product $\left(D_{1}^{\prime}, 0\right) \times\left(\mathbb{C}^{n-k}, 0\right)$, where $\left(D_{1}^{\prime}, 0\right) \subseteq\left(\mathbb{C}^{k}, 0\right)$ (and similarly for $\left.D_{2}\right)$.

Example 2.4. (1) Let $(D, 0)$ be the divisor in $\left(\mathbb{C}^{2}, 0\right)$ defined by $h_{1} h_{2}=x\left(y-x^{2}\right)$. Since $D$ has normal crossings at the origin, $D$ is splayed.

(2) The divisor $D=\left\{\left(x-y^{2}\right) z w=0\right\}$ is splayed in $\left(\mathbb{C}^{4}, 0\right)$ but its splayed components are not unique, for example $h_{1}=x-y^{2}$ and $h_{2}=z w$ or $h_{1}=\left(x-y^{2}\right) w$ and $h_{2}=z$.

(3) The divisor $D=\left\{\left(x z\left(x+z-y^{2}\right)=0\right\}\right.$ is not splayed in $\left(\mathbb{C}^{3}, 0\right)$.

Remark 2.5. The concept of splayed divisors was also introduced by J. Damon in the context of free divisors under the name of product union (see [Dam96]). In the theory of hyperplane arrangements one studies the analogous concept, under various names: e.g., in [OT92] splayed arrangements are called reducible and in [Bud12] they are referred to as decomposable.

\section{$\S 3$. Criteria for splayed divisors}

Let $(D, p)$ be a divisor in $(S, p)$. If the decomposition into irreducible components of $D$ is known, then there are effective methods to test whether $D$ has normal crossings at $p$ (see [Bod04]). The idea here is to find linearly independent tangent vectors, that is, to linearize the problem and thus reduce it to linear algebra. The algorithm of Bodnár does not work in order to test whether $(D, p)$ is splayed, since the irreducible components may be singular themselves at $p$.

In Proposition 3.3 it is shown that $D=D_{1} \cup D_{2}=\{g h=0\}$ is splayed at $p$ if and only if, up to multiplying $g, h$ with units, the Jacobian ideal $J_{g h}$ of $(D, p)$ is equal to $g J_{h}+h J_{g}$. This property can be tested in computer algebra systems like Singular. The second characterization is more geometrical. In Proposition 3.5 we show that for a splayed divisor Sing $D$ is the scheme-theoretic union of the intersection of $D_{1}$ and $D_{2}$, and of the singular loci Sing $D_{1}$ and $\operatorname{Sing} D_{2}$. The third criterion (Proposition 3.10) is nearer to Bodnár's normal crossings test. Therefore 
we use the notion of logarithmic derivations and differential forms (in the sense of K. Saito [Sai80]), which will be the main ingredient in the section about freeness of splayed divisors.

\section{§3.1. Jacobian ideal characterization 1-algebra}

Here a characterization of splayedness by Jacobian ideals is shown, which can be easily checked in concrete examples.

Definition 3.1. Let $D_{1}=\{g=0\}, D_{2}=\{h=0\}$ and $D=\{g h=0\}$ at $p$ be defined as above. We say that $J_{g h}$ has the Leibniz property if

$$
J_{g h}=g J_{h}+h J_{g}
$$

If $D$ is splayed then it is clear that its Jacobian ideal has the Leibniz property. To establish the other implication, first an intermediate ideal-theoretic characterization of splayedness is proven (Lemma 3.2).

Lemma 3.2. Let $\operatorname{dim} S=n$ and at a point $p=\left(x_{1}, \ldots, x_{n}\right)$ denote by $\mathcal{O}=$ $\mathcal{O}_{S, p}=\mathbb{C}\left\{x_{1}, \ldots, x_{n}\right\}$ the local ring at $p$. Let $D_{1}=\{g(x)=0\}, D_{2}=\{h(x)=0\}$ and $D=\{g h(x)=0\}$ be divisors, where we assume that $g, h \in \mathcal{O}$ are reduced and have no common factors. Then $\left.(D, p)=\left(D_{1}, p\right) \cup D_{2}, p\right)$ is splayed if and only if

$$
(g) \cap\left((g h)+J_{g h}\right)=g\left((h)+J_{h}\right) .
$$

Proof. First note that the inclusion $(g) \cap\left((g h)+J_{g h}\right) \subseteq g\left((h)+J_{h}\right)$ always holds since $g$ and $h$ have no common factors.

If $D$ is splayed, we can suppose without loss of generality that $D_{1}=$ $\{g(x, 0)=0\}$ and $D_{2}=\{h(0, y)=0\}$ where $(x, y)=\left(x_{1}, \ldots, x_{k}, y_{k+1}, \ldots, y_{n}\right)$. Then it is easy to see, via the Leibniz property of $J_{g h}$, that $g\left((h)+J_{h}\right)$ is contained in $(g) \cap\left((g h)+J_{g h}\right)$.

For the other direction, suppose that (3.1) holds. In this case it is easy to see that (3.1) is preserved under local isomorphisms of $(S, p)$. One may assume without loss of generality that $h\left(x_{1}, \ldots, y_{n}\right)=h\left(0, \ldots, 0, y_{k+1}, \ldots, y_{n}\right)$ and that

$$
\partial_{y_{i}} h \notin\left(h, \partial_{y_{k+1}} h, \ldots, \widehat{\partial_{y_{i}}} h, \ldots, \partial_{y_{n}} h\right)
$$

for all $i \in\{k+1, \ldots, n\}$. (If not so, suppose that e.g. $\partial_{x_{1}} h$ is contained in $\left(h, \partial_{x_{2}} h, \ldots, \partial_{x_{n}} h\right)$. Then by the triviality lemma there exists a locally biholomorphic map $\varphi:(S, p) \rightarrow(S, p)$ such that $h \circ \varphi(x)=v(x) h\left(0, x_{2}, \ldots, x_{n}\right)$, with $v \in \mathcal{O}^{*}$. Then set $\widetilde{h}:=h\left(0, x_{2}, \ldots, x_{n}\right)$ and $\widetilde{g}:=g \circ \varphi$. The divisor defined by $\widetilde{g} \cdot \widetilde{h}$ is clearly isomorphic to $D$, and similarly $D_{1}$ and $D_{2}$ are defined by $\widetilde{g}$ and $\widetilde{h}$.)

Now let $h(y)=h\left(0, \ldots, 0, y_{k+1}, \ldots, y_{n}\right)$ be such that (3.2) holds. From (3.1) it follows that $g\left(\partial_{y_{i}} h\right)$ is contained in $\left((g h)+J_{g h}\right)$ and by definition $g \partial_{y_{i}} h+h \partial_{y_{i}} g$ 
is also contained in this ideal. Hence $h\left(\partial_{y_{i}} g\right) \in\left((g h)+J_{g h}\right)$ for all $k+1 \leq i \leq n$. Thus we can write

$$
h\left(\partial_{y_{i}} g\right)=a_{i} g h+\sum_{j} a_{i j}\left(g \partial_{y_{j}} h+h \partial_{y_{j}} g\right)+\sum_{j} b_{i j} h \partial_{x_{j}} g
$$

where the summation indices run over the valid range. This equation can be rearranged as

$$
h\left(\partial_{y_{i}} g-a_{i} g-\sum_{j} a_{i j} \partial_{y_{j}} g-\sum_{j} b_{i j} \partial_{x_{j}} g\right)=g \sum_{j} a_{i j}\left(\partial_{y_{j}} h\right) .
$$

Since $g$ and $h$ have by assumption no common factors, we conclude from (3.3) that $\partial_{y_{i}} g-a_{i} g-\sum_{j} a_{i j} \partial_{y_{j}} g-\sum_{j} b_{i j} \partial_{x_{j}} g \in(g)$ and that $\sum_{j} a_{i j}\left(\partial_{y_{j}} h\right)=c_{i} h$ for any $k+1 \leq i \leq n$ and some $c_{i} \in \mathcal{O}$. Then (3.2) implies that any $a_{i j} \in \mathfrak{m}$ : if this were not the case, that is, if some $a_{i m}$ is in $\mathcal{O}^{*}$, then via the equation $\sum_{j} a_{i j}\left(\partial_{y_{j}} h\right)=c_{i} h$ one can express $\partial_{y_{m}} h$ in terms of $h$ and the other $\partial_{y_{j}} h$, which contradicts (3.2). Hence it follows that

$$
\partial_{y_{i}} g \in\left(g, \partial_{x_{1}} g, \ldots, \partial_{x_{k}} g\right)+\mathfrak{m}\left(\partial_{y_{k+1}} g, \ldots, \partial_{y_{n}} g\right)
$$

for any $k+1 \leq i \leq n$. By an application of Nakayama's lemma we obtain

$$
\partial_{y_{i}} g \in\left(g, \partial_{x_{1}} g, \ldots, \partial_{x_{k}} g\right) \text { for all } i=k+1, \ldots, n .
$$

By the triviality lemma there exists a locally biholomorphic $\psi:(S, p) \rightarrow(S, p)$ with $\psi(x, y)=\left(\psi_{1}(x, y), \ldots, \psi_{k}(x, y), y_{k+1}, \ldots, y_{n}\right)$ such that $g \circ \psi$ is equal to $v g\left(x_{1}, \ldots, x_{k}, 0\right)$ for a unit $v \in \mathcal{O}$. Set $\tilde{g}:=v^{-1}(g \circ \psi)$ and $\tilde{h}:=h \circ \psi=h=$ $h\left(0, \ldots, 0, y_{k+1}, \ldots, y_{n}\right)$. By construction $\tilde{g} \tilde{h}$ defines a splayed divisor that is isomorphic to $D$.

Proposition 3.3. Let $(S, D)$ be a complex manifold $S$ with $\operatorname{dim} S=n$, together with a divisor $D \subseteq S$ that is locally at a point defined by $g h=0$, where $g$ and $h$ are reduced elements of $\mathcal{O}_{S, p}$ that are not necessarily irreducible but have no common factor. Then $D=\{g=0\} \cup\{h=0\}$ is splayed at $p$ if $J_{g h}$ has the Leibniz property

$$
J_{g h}=g J_{h}+h J_{g} .
$$

Conversely, if $D=\{g h=0\}$ is splayed and $g$ and $h$ are chosen in different variables, then $J_{g h}$ has the Leibniz property. This means that up to possible multiplication of $g$ and $h$ with units, $J_{g h}$ has the Leibniz property.

Proof. As already remarked, if $D$ is splayed then we can choose the defining equations $g$ and $h$ in separated variables and it is clear that $J_{g h}=g J_{h}+h J_{g}$. Conversely, it is enough to show the equality $(g) \cap\left((g h)+J_{g h}\right)=g\left((h)+J_{h}\right)$ from Lemma 3.2. 
Since $(g) \cap\left((g h)+J_{g h}\right)$ is always contained in $g\left((h)+J_{h}\right)$ it remains to check the other inclusion. Take a $\beta \in g\left((h)+J_{h}\right)$. Then a straightforward calculation shows that $\beta$ is also contained in $(g) \cap\left((g h)+J_{g h}\right)$.

As pointed out by P. Aluffi, $D=\{g h=0\}$ is splayed if and only if

$$
(g h)+J_{g h}=(g h)+g J_{h}+h J_{g},
$$

regardless of multiplying $g$ and $h$ by units. Equation (3.4) can also easily be shown with Lemma 3.2. For a different approach to this characterization of splayedness, see $[\mathrm{AF} 12]$.

Example 3.4. Let $D$ be the divisor in $\mathbb{C}^{3}$ given at a point $p$ by $x\left(x+y^{2}-z^{3}\right)$. Then $D$ is the union of two smooth components $D_{1}=\left\{h=x+y^{2}-z^{3}=0\right\}$ and $H=\{g=x=0\}$. The ideal $(g h)+J_{g h}=J_{g h}=\left(2 x+y^{2}-z^{3}, x y, x z^{2}\right)$ is strictly contained in $(g h)+g J_{h}+h J_{g}=g J_{h}+h J_{g}=\left(x, y^{2}-z^{3}\right)$. Thus $D$ is not a splayed divisor (see Figure 1).

\section{§3.2. Jacobian ideal characterization 2-geometry}

Now we characterize a splayed divisor $D \subseteq S$, locally at a point $p$ given by a $g h \in \mathcal{O}_{S, p}$, by $\mathcal{O}_{\text {Sing } D, p}=\mathcal{O}_{S, p} /\left((g h)+J_{g h}\right)$. This characterization reflects the geometry of $(D, p)$, namely the two splayed components meeting transversally.

Proposition 3.5. The divisor $D=D_{1} \cup D_{2}$, defined at $p$ as above, is splayed if and only if

$$
\left((g h)+J_{g h}\right)=(g, h) \cap\left((g)+J_{g}\right) \cap\left((h)+J_{h}\right) .
$$

Proof. Recall here from Lemma 3.2 that a divisor $\{g h=0\}$ is splayed if and only if (3.1) holds. First suppose that (3.5) holds. A straightforward calculation shows

For the other implication we use Grauert's division theorem (for the notation and statement see [dJP00, Theorem 7.1.9]): Suppose that $D=\{g h=0\}$ is splayed. Then without loss of generality $g(x, y)=g(x)$ and $h(x, y)=h(y)$ in $\mathbb{C}\left\{x_{1}, \ldots, x_{k}, y_{k+1}, \ldots, y_{n}\right\}$. Clearly $\left((g h)+J_{g h}\right) \subseteq(g, h) \cap\left((g)+J_{g}\right) \cap\left((h)+J_{h}\right)$. So let $\alpha$ be an element of the right-hand side, that is, $\alpha=a g+b h=c g+\sum_{i=1}^{k} a_{i} \partial_{x_{i}} g$ for some $a, b, c, a_{i} \in \mathcal{O}$. Then also $\alpha-a g=b h=(c-a) g+\sum_{i=1}^{k} a_{i} \partial_{x_{i}} g$ is contained in $\left((g)+J_{g}\right)$. By Grauert's division theorem there exist some $\tilde{a}, \tilde{a}_{i}, r, \tilde{r}_{i}$ such that for all $i=1, \ldots, k$ one has $c-a=\tilde{a} h+r$ and $a_{i}=\tilde{a}_{i} h+r_{i}$ and the leading monomial $L(h)$ does not divide any monomial of the unique remainders $r, r_{i}$. Then 
write

$$
\left(b-\tilde{a} g-\sum_{i=1}^{k} \tilde{a}_{i}\left(\partial_{x_{i}} g\right)\right) h=r g+\sum_{i=1}^{k} r_{i}\left(\partial_{x_{i}} g\right) .
$$

Since $h$ only depends on $y$ and $g$ only on $x$, it follows that $L(h)$ also does not divide any of the monomials of the right-hand side of the equation. But this is only possible if $\left(b-\tilde{a} g-\sum_{i=1}^{k} \tilde{a}_{i}\left(\partial_{x_{i}} g\right)\right) h=0$. It follows that $b$ is contained in $\left((g)+J_{g}\right)$. Interchanging the roles of $g$ and $h$ yields that $a \in\left((h)+J_{h}\right)$ and thus $\alpha \in\left(g h, g J_{h}+h J_{g}\right)$. The Leibniz property of $J_{g h}$ implies that $\alpha \in\left((g h)+J_{g h}\right)$.

Remark 3.6. Here it can be seen that for splayed divisors the Jacobian ideal is the intersection of the two ideals defining the singular loci of the splayed components $D_{1}$ and $D_{2}$ plus the intersection of $D_{1}$ and $D_{2}$. For two smooth divisors $D_{1}$ and $D_{2}$ this means that $D=D_{1} \cup D_{2}$ is a splayed divisor if and only if the schemetheoretical intersection $D_{1} \cap D_{2}$ is smooth, which is in turn equivalent to saying that $D_{1}$ and $D_{2}$ intersect transversally (cf. [Li09, FH10]). Note that for varieties of arbitrary codimension the condition that the scheme-theoretical intersection is smooth, leads to the notion of clean intersection (see [Li09]).

Example 3.7. Let $D \subseteq \mathbb{C}^{3}$ be given at the origin by $g h=x\left(x+y^{2}+z^{3}\right)=0$. Then $D$ is not splayed at the origin. Here the intersection of the two components is given by the ideal $(g, h)=\left(x, y^{2}+z^{3}\right)$. Also consider the divisor $D^{\prime} \subseteq \mathbb{C}^{3}$ that is given by $g^{\prime} h^{\prime}=x\left(y^{2}+z^{3}\right)$. Clearly $D^{\prime}$ is splayed at the origin and the intersection of the two components is given by the ideal $\left(g^{\prime}, h^{\prime}\right)=\left(x, y^{2}+z^{3}\right)$ (see Figure 1).

\section{§3.3. Logarithmic derivation characterization-tangency}

As already mentioned in the introduction, two submanifolds of a manifold intersect transversally at a point if their respective tangent spaces at that point together generate the tangent space of the ambient manifold. In this section we will translate this definition to splayed divisors with the help of logarithmic derivations (Proposition 3.10). Logarithmic derivations were introduced by K. Saito in [Sai80] and are a useful tool in studying tangent behaviour for singular varieties. They lead to the definition of free divisors, which will be studied in more detail in Section 4.1.

3.3.1. A very brief recap of Saito's theory of free divisors ([Sai80]). Let $D$ be a divisor in $S$ defined at $p$ by $D=\{h=0\}$. A logarithmic vector field (or logarithmic derivation) (along $D$ ) is a holomorphic vector field on $S$, that is, an element of $\operatorname{Der}_{S}$, satisfying one of the two equivalent conditions:

(i) For any smooth point $p$ of $D$, the vector $\delta(p)$ of $p$ is tangent to $D$,

(ii) For any point $p$, where $(D, p)$ is given by $h=0$, the germ $\delta(h)$ is contained in the ideal $(h)$ of $\mathcal{O}_{S, p}$. 
The module of germs of logarithmic derivations (along $D$ ) at $p$ is denoted by

$$
\operatorname{Der}_{S, p}(\log D)=\left\{\delta: \delta \in \operatorname{Der}_{S, p} \text { such that } \delta h \in(h)\right\},
$$

The $\operatorname{Der}_{S, p}(\log D)$ are the stalks at points $p$ of the sheaf $\operatorname{Der}_{S}(\log D)$ of $\mathcal{O}_{S^{-}}$ modules. Similarly we define logarithmic differential forms: a meromorphic $q$-form $\omega$ is logarithmic (along $D$ ) at a point $p$ if $\omega h$ and $h d \omega$ are holomorphic in an open neighbourhood around $p$. We write

$$
\Omega_{S, p}^{q}(\log D)=\{\omega: \omega \text { germ of a logarithmic } q \text {-form at } p\}
$$

One can show that $\operatorname{Der}_{S, p}(\log D)$ and $\Omega_{S, p}^{1}(\log D)$ are reflexive $\mathcal{O}_{S, p}$-modules dual to each other (see [Sai80]). One says that $(D, p)$ is free or that $D$ is free at $p$ if $\operatorname{Der}_{S, p}(\log D)$ resp. $\Omega_{S, p}^{1}(\log D)$ is a free $\mathcal{O}_{S, p}$-module. In Section 4.1 free divisors are discussed in more detail and the following theorem is used, which makes it possible to test whether $D$ is free (cf. [Sai80, Thm. 1.8]):

Theorem 3.8 (Saito's criterion). Let $(S, D), p$ and $h$ be as defined in the introduction. The $\mathcal{O}_{S, p}$-module $\operatorname{Der}_{S, p}(\log D)$ is free if and only if there exist $n$ vector fields $\delta_{i}=\sum_{j=1}^{n} a_{i j}(x) \partial_{x_{j}}$ in $\operatorname{Der}_{S, p}(\log D), i=1, \ldots, n$, such that $\operatorname{det}\left(a_{i j}(x)\right)$ is equal to $h$ up to an invertible factor. Moreover, the vector fields $\delta_{1}, \ldots, \delta_{n}$ then form a basis for $\operatorname{Der}_{S, p}(\log D)$.

Remark 3.9. The module $\operatorname{Der}_{S, p}(\log D)$ naturally carries the structure of a Lie algebra. It was considered under the name of tangent algebra in [HM93], where also many properties of algebraic and analytic varieties were characterized in terms of tangent algebras. The Lie algebra structure of logarithmic derivations is also considered in [GS09, Sek08].

\subsubsection{Logarithmic derivations and splayed divisors}

Proposition 3.10. Let $(D, p)=\left(D_{1}, p\right) \cup\left(D_{2}, p\right) \subseteq(S, p) \cong\left(\mathbb{C}^{n}, 0\right)$ be a divisor such that $\left(D_{1}, p\right)=\{g=0\}$ and $\left(D_{2}, p\right)=\{h=0\}$ for some $g$, $h$ not necessarily irreducible but with no common factor. Then $D$ is splayed at $p$ if and only if

$$
\operatorname{Der}_{S, p}\left(\log D_{1}\right)+\operatorname{Der}_{S, p}\left(\log D_{2}\right)=\operatorname{Der}_{S, p} .
$$

Proof. As above, we denote by $(x, y):=\left(x_{1}, \ldots, x_{k}, y_{k+1}, \ldots, y_{n}\right)$ the coordinates at $p$ and by $\mathcal{O}=\mathbb{C}\{x, y\}$ the corresponding local ring. If $D$ is splayed then without loss of generality one has $g=g(x, 0)$ and $\operatorname{Der}_{S, p}\left(\log D_{1}\right)$ is generated by some $\delta_{1}, \ldots, \delta_{m}, m \geq k, \partial_{y_{k+1}}, \ldots, \partial_{y_{n}}$. Similarly $h=h(0, y)$ and $\operatorname{Der}_{S, p}\left(\log D_{2}\right)$ is generated by some $\partial_{x_{1}}, \ldots, \partial_{x_{k}}, \varepsilon_{1}, \ldots, \varepsilon_{l}$ for some $l \geq n-k$. Then clearly we have

$$
\operatorname{Der}_{S, p}\left(\log D_{1}\right)+\operatorname{Der}_{S, p}\left(\log D_{2}\right)=\mathcal{O}\left\langle\partial_{x_{1}}, \ldots, \partial_{x_{k}}, \partial_{y_{k+1}}, \ldots, \partial_{y_{n}}\right\rangle=\operatorname{Der}_{S, p} .
$$


For the other implication we may assume that $g(x, y)=g(x, 0)$ and

$$
\partial_{x_{i}} g \notin\left(\partial_{x_{1}} g, \ldots, \widehat{\partial_{x_{i}} g}, \ldots, \partial_{x_{k}} g\right)
$$

for all $i \leq k$ by a similar argument to Lemma 3.2. Then it is clear that $\operatorname{Der}_{S, p}\left(\log D_{1}\right)$ can be generated by some $\delta_{1}, \ldots, \delta_{m}$ and $\partial_{y_{k+1}}, \ldots, \partial_{y_{n}}$, where $m \geq k$ and the coefficients of the $\delta_{i}$ only depend on $x_{1}, \ldots, x_{k}$ and lie in the maximal ideal $\mathfrak{m} \subseteq \mathcal{O}$.

Using $\partial_{x_{i}} \in \mathcal{O}\left\langle\varepsilon_{1}, \ldots, \varepsilon_{l}, \delta_{1}, \ldots, \delta_{m}, \partial_{y_{k+1}}, \ldots, \partial_{y_{n}}\right\rangle$ and computing $\partial_{x_{i}} h$ we obtain (similar to the proof of Lemma 3.2) that

$$
\left(\partial_{x_{1}} h, \ldots, \partial_{x_{k}} h\right) \subseteq\left(h, \partial_{y_{k+1}} h, \ldots, \partial_{y_{n}} h\right) .
$$

An application of the triviality lemma shows that $D_{2}$ can be chosen independently of the variables $x_{1}, \ldots, x_{k}$, and thus $D$ is splayed.

In view of Proposition 3.10 and the duality of $\operatorname{Der}_{S, p}(\log D)$ and $\Omega_{S, p}^{1}(\log D)$ we ask:

Question 3.11. Can one express the splayedness of a divisor $\left(D_{1} \cup D_{2}, p\right)$ in terms of $\Omega_{S, p}^{1}\left(\log D_{1}\right), \Omega_{S, p}^{1}\left(\log D_{2}\right)$ and $\Omega_{S, p}^{1}$ ?

\section{§4. Applications}

\section{$\S 4.1$. Free divisors and normal crossings}

In some sense free divisors are a generalization of normal crossing divisors: their modules of tangent vector fields are free. However, irreducible (non-smooth) free divisors are highly singular, that is, they are non-normal and their singular loci are Cohen-Macaulay of codimension 1 in the divisor (see [Ale90, Sim06]). Here we consider the relationship between free and splayed divisors and in particular between splayed and normal crossing divisors. First we show that a splayed divisor is free if and only if its splayed components are free (Proposition 4.1). Then we turn to normal crossing divisors, in particular to the problem of characterizing normal crossing divisors by their Jacobian ideal. This problem was stated by H. Hauser and considered in [Fab11], where a general answer was found. Here we use the ideal-theoretic characterizations of splayed divisors in order to show that a divisor consisting of smooth irreducible components has normal crossings at a point $p$ if and only if it is free at $p$ and its Jacobian ideal is radical (Corollary 4.7).

First let us describe the structure of $\operatorname{Der}_{S, p}(\log D)$ for splayed divisors in a different way. Let $S, T$ be complex manifolds of dimensions $n, m$ and suppose that $(S \times T, 0) \cong\left(\mathbb{C}^{n+m}, 0\right)$, with complex coordinates $(x, y)=\left(x_{1}, \ldots, x_{n}, y_{1}, \ldots, y_{m}\right)$ 
at the origin. Let $\left(D_{1}^{x}, 0\right)$ be a divisor in $(S, 0)$, which is defined by a reduced $g^{\prime}(x) \in \mathcal{O}_{S, 0} \cong \mathbb{C}\left\{x_{1}, \ldots, x_{n}\right\}$ and which has a logarithmic derivation module over $\mathbb{C}\{x\}$ denoted by $\operatorname{Der}_{S, 0}\left(\log D_{1}^{x}\right)$. Then we may consider the cylinder over $D_{1}^{x}$ in the $T$-direction in $(S \times T, 0)$, namely the hypersurface $D_{1}$ defined by $g(x, y)=$ $g(x, 0):=g^{\prime}(x) \in \mathbb{C}\{x, y\}$. It is easy to see that

$$
\operatorname{Der}_{S \times T, 0}\left(\log D_{1}\right)=\left(\operatorname{Der}_{S, 0}\left(\log D_{1}^{x}\right) \otimes_{\mathbb{C}\{x\}} \mathbb{C}\{x, y\}\right) \oplus\left(\operatorname{Der}_{T, 0} \otimes_{\mathbb{C}\{y\}} \mathbb{C}\{x, y\}\right) .
$$

Similarly define $D_{2}^{y}, D_{2}$ with equations $h^{\prime}(y)=h(x, y)$ and $\operatorname{Der}_{S \times T, 0}\left(\log D_{2}\right)$. Thus we define the (splayed) divisor $D=D_{1} \cup D_{2}$ in $S \times T$ that is given at 0 by the equation $g h=0$. Since $g$ and $h$ have separated variables, there is a natural splitting of $\operatorname{Der}_{S \times T, 0}(\log D)$ :

$$
\begin{aligned}
& \operatorname{Der}_{S \times T, 0}(\log D) \\
& =\left(\operatorname{Der}_{S, 0}\left(\log D_{1}^{x}\right) \otimes_{\mathbb{C}\{x\}} \mathbb{C}\{x, y\}\right) \oplus\left(\operatorname{Der}_{T, 0}\left(\log D_{2}^{y}\right) \otimes_{\mathbb{C}\{y\}} \mathbb{C}\{x, y\}\right) .
\end{aligned}
$$

Proposition 4.1. Let $(D, p)=\left(D_{1}, p\right) \cup\left(D_{2}, p\right)$ be a splayed divisor in $S \cong$ $\mathbb{C}^{n+m}$ defined as above. The divisor $D=\{g(x) h(y)=0\}$ is free if and only if $D_{1}=\{g(x)=0\}$ and $D_{2}=\{h(y)=0\}$ are both free.

Proof. If both $D_{1}$ and $D_{2}$ are free then there exist bases of $\operatorname{Der}_{S \times T, p}\left(\log D_{1}\right)$ and $\operatorname{Der}_{S \times T, p}\left(\log D_{2}\right)$ of the form

$$
\delta_{1}=\sum_{i=1}^{n} a_{1 i} \partial_{x_{i}}, \ldots, \delta_{n}=\sum_{i=1}^{n} a_{n i} \partial_{x_{i}}, \delta_{n+1}=\partial_{y_{1}}, \ldots, \delta_{n+m}=\partial_{y_{m}}
$$

and

$$
\varepsilon_{1}=\partial_{x_{1}}, \ldots, \varepsilon_{n}=\partial_{x_{n}}, \varepsilon_{n+1}=\sum_{i=1}^{m} b_{n+1, i} \partial_{y_{i}}, \ldots, \varepsilon_{n+m}=\sum_{i=1}^{m} b_{n+m, i} \partial_{y_{i}} .
$$

It is easy to see that any $\delta_{i}$ for $1 \leq i \leq n$ and any $\varepsilon_{j}$ for $n+1 \leq j \leq n+m$ is also an element of $\operatorname{Der}_{S \times T, p}(\log D)$. By Saito's criterion (Thm. 3.8) it follows that $\delta_{1}, \ldots, \delta_{n}, \varepsilon_{n+1}, \ldots, \varepsilon_{n+m}$ form a basis of $\operatorname{Der}_{S \times T, p}(\log D)$.

Conversely, suppose $\operatorname{Der}_{S \times T, p}(\log D)$ is free. Since $\operatorname{Der}_{S \times T, p}(\log D)$ is free, it follows by (4.1) that $\operatorname{Der}_{S, 0}\left(\log D_{1}^{x}\right) \otimes_{\mathbb{C}\{x\}} \mathbb{C}\{x, y\}$ and $\operatorname{Der}_{T, 0}\left(\log D_{2}^{y}\right) \otimes_{\mathbb{C}\{y\}} \mathbb{C}\{x, y\}$ are projective $\mathcal{O}_{S \times T, p}$-modules. Since the notions of projective and free module over regular local rings coincide, these two modules are even free.

4.1.1. Radical Jacobian ideal — normal crossings. In [Fab11] the following problem (proposed by H. Hauser) was considered: suppose that $D \subseteq S, \operatorname{dim} S=n$, is a divisor that is locally at a point $p \in S$ given by $\{h=0\}$. Can we determine if $D$ has normal crossings at $p$ from the knowledge of its Jacobian ideal $J_{h}$ ? 
It was shown that $D$ has normal crossings in case $D$ is free, the Jacobian ideal is radical and the normalization of $D$ is smooth (see [Fab12, Thm. 2.4]).

Here we use splayed divisors to show this when $(D, p)=\bigcup_{i=1}^{m}\left(D_{i}, p\right)$ is a union of smooth irreducible components $D_{i}$. Note that in this special case the hypothesis on the normalization is not needed. Moreover, the problem can be reduced to the case where $(D, p)$ is irreducible (Proposition 4.6).

Remark 4.2. One can show, using the theorem of Briançon-Skoda, that $J_{h}$ radical implies that $h$ is already contained in $J_{h}$ (see [Fab12]).

Lemma 4.3. Let $D=\{g h=0\}$ be splayed at $p=\left(x_{1}, \ldots, x_{k}, y_{k+1}, \ldots, y_{n}\right)$ in $S \cong \mathbb{C}^{n}$ and denote by $D_{1}=\{g=g(x, 0)=0\}$ and $D_{2}=\{h=h(0, y)=0\}$ its splayed components. The Jacobian ideal of $D$, denoted by $J_{g h}$, is radical if and only if both $J_{h}$ and $J_{g}$ are radical.

Proof. Suppose that $J_{g}$ and $J_{h}$ are radical. Similarly to the proof of Proposition 3.5 one concludes that the ideals $J_{g h}$ and $(g, h) \cap J_{g} \cap J_{h}$ are equal. We compute the radical

$$
\sqrt{J_{g h}}=\sqrt{(g, h) \cap J_{g} \cap J_{h}}=\sqrt{(g, h)} \cap \sqrt{J_{g}} \cap \sqrt{J_{h}}=(g, h) \cap J_{g} \cap J_{h}=J_{g h},
$$

where the third equality holds because of our assumptions.

Conversely, suppose that $J_{g h}=\sqrt{J_{g h}}$. Then $g h$ is an element of $J_{g h}$ (cf. Remark 4.2). Localization of $\mathbb{C}\{x, y\}$ in $g$ yields $\left(J_{g h}\right)_{g}=\left((h)+J_{h}\right)_{g}$, which is radical, since $J_{g h}$ is radical. Note that for an ideal $I \subseteq \mathbb{C}\{x, y\}$, we denote by $I_{g}$ the localization of $I$ in $g$ (cf. [Mat86]). Using the fact that $\left((h)+J_{h}\right)_{g}$ is an intersection of prime ideals in the localization, a direct computation shows that any element of $\sqrt{\left((h)+J_{h}\right)}$ is already contained in $\left((h)+J_{h}\right)$. Similarly one proves $\left((g)+J_{g}\right)=\sqrt{\left((g)+J_{g}\right)}$.

Lemma 4.4. Let $D \subseteq S$ be a divisor given at $p \in S$ by $\{g h=0\}$ with $g h \in \mathcal{O}_{S, p}$ reduced and suppose that $J_{g h}$ is radical. Then

$$
J_{g h}=g J_{h}+h J_{g}
$$

In particular, $D=D_{1} \cup D_{2}$ defined by $D_{1}=\{g=0\}$ and $D_{2}=\{h=0\}$ is splayed at $p$.

Proof. Consider the element $g \partial_{x_{i}}(g h)$, which is contained in $J_{g h}$ for any $i=$ $1, \ldots, n$. Since $J_{g h}$ is radical, $g h$ is contained in $J_{g h}$, which forces $g^{2}\left(\partial_{x_{i}} h\right)$ to be in $J_{g h}$. Using the fact that $J_{g h}$ is an intersection of prime ideals $\mathfrak{p}_{j}, j=1, \ldots, k$, one deduces that either $g$ or $\partial_{x_{i}} h$ is contained in any $\mathfrak{p}_{\mathbf{j}}$. Hence $g\left(\partial_{x_{i}} h\right) \in J_{g h}$. This 
yields

$$
J_{g h}=\left(g \partial_{x_{1}}(h), \ldots, g \partial_{x_{n}}(h), h \partial_{x_{1}}(g), \ldots, h \partial_{x_{n}}(g)\right)=g J_{h}+h J_{g}
$$

By Proposition 3.3, $(D, p)$ is splayed.

Example 4.5. Splayed divisors need not have radical Jacobian ideals, as the following example shows. Let $D$ be the divisor in $\left(\mathbb{C}^{3}, 0\right)$ with coordinates $(x, y, z)$ at 0 , that is defined by $g h=x\left(y^{2}+z^{3}\right)$. Then clearly $(D, 0)$ is splayed. The Jacobian ideal is $J_{g h}=\left(y^{2}+z^{3}, x y, x z^{2}\right)=\left(y, z^{2}\right) \cap\left(x, y^{2}+z^{3}\right)$, which is not radical. Note that $D$ is a free divisor.

Proposition 4.6. Let $D=D_{1} \cup D_{2}$ be a divisor in an $n$-dimensional complex manifold $S$ and let $D, D_{1}$ and $D_{2}$ at a point $p \in S$ be defined by the equations $g h$, $g$ and $h$, respectively. Suppose that $J_{g h}$ is radical. Then $D$ is splayed and $J_{h}$ and $J_{g}$ are also radical. If moreover $D$ is free at $p$ then also $D_{1}$ and $D_{2}$ are free at $p$.

Proof. This follows from Proposition 3.3, Proposition 4.1 and Lemma 4.4.

Corollary 4.7. Let $(S, D)$ be a complex manifold with $\operatorname{dim} S=n$, together with a divisor $D \subseteq S$, and suppose that locally at a point $p \in S$ the divisor $(D, p)$ has a decomposition $\bigcup_{i=1}^{m}\left(D_{i}, p\right)$ into irreducible components such that each $\left(D_{i}, p\right)$ is smooth. Let the corresponding equation of $D$ at $p$ be $h=h_{1} \cdots h_{m}$. If $D$ is free at $p$ and $J_{h}=\sqrt{J_{h}}$ then $D$ has normal crossings at $p$.

Proof. We use induction on $n$. If $n=2$, then an explicit computation or an application of the theorem of Mather-Yau ([MY82]) shows the assertion. Now suppose the assertion is true for divisors in manifolds of dimension $n-1$. For a smooth component $D_{1}$ of $D$, one can find local coordinates $\left(x_{1}, \ldots, x_{n}\right)$ such that $D_{1}=\left\{x_{1}=0\right\}$. Proposition 4.6 shows that the divisor $\left(D \backslash D_{1}\right):=h_{2} \cdots h_{m}$ is also free and has a radical Jacobian ideal. Moreover, $D$ is locally splayed, that is, $D \backslash D_{1}$ is locally isomorphic to some divisor depending only on the last $n-1$ coordinates. Thus by induction hypothesis $D \backslash D_{1}$ is isomorphic to a normal crossing divisor $y_{2} \cdots y_{m}=0$, where the $y_{i}$ are the result of a coordinate transformation of $\left(x_{1}, \ldots, x_{n}\right)$ such that $x_{1}=y_{1}$. This implies that $m \leq n-1$. Hence $D$ is isomorphic to the normal crossing divisor $x_{1} y_{2} \cdots y_{m}$.

\section{$\S 4.2$. Hilbert-Samuel polynomials}

Splayed divisors are particularly interesting for computational reasons. We start here a study of properties of splayed divisors by considering their Hilbert-Samuel polynomials. We find that multiplicities behave the same for splayed as for nonsplayed divisors but that the Hilbert-Samuel polynomials for splayed divisors are 
additive, which means the following: Let $(D, p)=\left(D_{1}, p\right) \cup\left(D_{2}, p\right)$ be a splayed divisor at a point $p$ in a complex manifold. Then from the exact sequence

$$
0 \rightarrow \mathcal{O}_{D, p} \rightarrow \mathcal{O}_{D_{1}, p} \oplus \mathcal{O}_{D_{2}, p} \rightarrow \mathcal{O}_{D_{1} \cap D_{2}, p} \rightarrow 0
$$

it follows that

$$
\chi_{D, p}+\chi_{D_{1} \cap D_{2}, p}=\chi_{D_{1}, p}+\chi_{D_{2}, p},
$$

where $\chi_{D, p}$ denotes the Hilbert-Samuel polynomial of $D$ at $p$. Since one can compute the Hilbert-Samuel polynomials of divisors in an easy way ([dJP00, Lemma 4.2.20]), this yields a method to compute $\chi_{D_{1} \cap D_{2}, p}$.

The Hilbert-Samuel function and the Hilbert-Samuel polynomial do not only depend on the module one is considering but also on a chosen filtration. However, the degree and leading coefficient of the Hilbert-Samuel polynomial are independent of the filtration. We use notation from [Mat86] and [dJP00].

Let $R$ be a noetherian local ring with maximal ideal $\mathfrak{m}$. Let $I \subseteq R$ be an ideal and let $M$ be a module over $R$. A set $\left\{M_{n}\right\}_{n \geq 0}$ of submodules of $M$ is called an $I$-filtration of $M$ if $M=M_{0} \supset M_{1} \supset M_{2} \supset \cdots$ and $I M_{n} \subseteq M_{n+1}$ for all $n \geq 0$.

In the following we will always suppose that $M$ is a finitely generated $R$ module. Let $\mathfrak{q}$ be an $\mathfrak{m}$-primary ideal of $R$ and let $\left\{M_{i}\right\}$ be a $\mathfrak{q}$-filtration. Then the Hilbert-Samuel function of the filtration $\left\{M_{i}\right\}$ is

$$
\mathrm{HS}_{\left\{M_{i}\right\}}: \mathbb{N} \rightarrow \mathbb{N}, \quad d \mapsto \text { length }_{R / \mathfrak{m}} M / M_{d}
$$

If $M_{i}=\mathfrak{m}^{i} M$ then we simply write $\mathrm{HS}_{M}$. One can show that there exists a polynomial $\chi_{\left\{M_{i}\right\}}$ with rational coefficients such that $\operatorname{HS}_{\left\{M_{i}\right\}}(d)=\chi_{\left\{M_{i}\right\}}(d)$ for $d$ sufficiently large. We call $\chi_{M}^{\mathfrak{q}}:=\chi_{\left\{\mathfrak{q}^{n} M\right\}_{n>0}}$ the Hilbert-Samuel polynomial of $M$ with respect to $\mathfrak{q}$. The degree $d$ of $\chi_{M}^{\mathfrak{q}}(k)=\sum_{i=0}^{d} a_{i} k^{i}$ only depends on $M$ and not on $\mathfrak{q}$.

When considering Hilbert-Samuel polynomials of modules over a local ring $\mathcal{O}=\mathbb{C}\left\{x_{1}, \ldots, x_{n}\right\}$ with respect to the maximal ideal $\mathfrak{m}$, one can use standard bases to simplify computations. For definitions and notation about standard bases we refer to [dJP00]. Here we only need the following facts:

Fact (i). Take the degree lexicographical ordering $<$. Then for an ideal $I \subseteq \mathcal{O}$ one has

$$
\mathrm{HS}_{\mathcal{O} / I}(k)=\operatorname{HS}_{\mathcal{O} / L(I)}(k),
$$

where $L(I)$ denotes the leading ideal of $I$. In particular, $\mathcal{O} / I$ and $\mathcal{O} / L(I)$ have the same Hilbert-Samuel polynomial with respect to $\mathfrak{m}$.

Fact (ii). Let $f, g \in \mathcal{O}$ and assume that $L(f)$ and $L(g)$ are coprime. Then $L((f, g))=(L(f), L(g))$, that is, $f, g$ are a standard basis of the ideal $(f, g)$. 
In contrast to the graded case, the Hilbert-Samuel polynomial is not additive on exact sequences: one has a certain error polynomial, whose degree can be determined with a theorem of Flenner and Vogel (see [FV93]):

Theorem 4.8 (Flenner-Vogel). Let $(R, \mathfrak{m})$ be a noetherian local ring, $0 \rightarrow M^{\prime} \rightarrow$ $M \rightarrow M^{\prime \prime} \rightarrow 0$ an exact sequence of finitely generated $R$-modules and $\mathfrak{q}$ an $\mathfrak{m}$-primary ideal. Denote further

$$
\operatorname{Gr}_{\mathfrak{q}}(N)=\bigoplus_{i=0}^{\infty} \mathfrak{q}^{i} N / \mathfrak{q}^{i+1} N
$$

the associated graded module of a finite $R$-module $N$. Then

(a) supp $\operatorname{ker}\left(\operatorname{Gr}_{\mathfrak{q}}\left(M^{\prime}\right) \rightarrow \operatorname{Gr}_{\mathfrak{q}}(M)\right)=\operatorname{supp} \operatorname{ker}\left(\operatorname{Gr}_{\mathfrak{q}}(M) / \operatorname{Gr}_{\mathfrak{q}}\left(M^{\prime}\right) \rightarrow \operatorname{Gr}_{\mathfrak{q}}\left(M^{\prime \prime}\right)\right)$.

(b) Denote by $d$ the dimension of these supports. Then there is a polynomial $S$ of degree $d-1$ such that for $n \gg 0$ we have

$$
S(n):=\chi_{M^{\prime}}^{\mathfrak{q}}(n)+\chi_{M^{\prime \prime}}^{\mathfrak{q}}(n)-\chi_{M}^{\mathfrak{q}}(n) .
$$

In particular, if $d=0$, then $S=0$.

Before turning to splayed divisors, consider the additivity problem of the Hilbert-Samuel polynomial for arbitrary finitely generated modules over a local ring $(R, \mathfrak{m})$. If

$$
0 \rightarrow N \rightarrow M \rightarrow M / N \rightarrow 0
$$

is an exact sequence of finitely generated $R$-modules and $\mathfrak{q}$ an $\mathfrak{m}$-primary ideal, then

$$
0 \rightarrow N /\left(\mathfrak{q}^{n} M \cap N\right) \rightarrow M / \mathfrak{q}^{n} M \rightarrow(M / N) / \mathfrak{q}^{n}(M / N) \rightarrow 0
$$

is an exact sequence, which implies

$$
\chi_{M}^{\mathfrak{q}}=\chi_{M / N}^{\mathfrak{q}}+\chi_{\left\{\mathfrak{q}^{n} M \cap N\right\}} .
$$

Here $\chi_{\left\{\mathfrak{q}^{n} M \cap N\right\}}$ denotes the Hilbert-Samuel polynomial with respect to the filtration $N /\left(\mathfrak{q}^{n} M \cap N\right)$ and in general one has $\chi_{N}^{\mathfrak{q}} \neq \chi_{\left\{\mathfrak{q}^{n} M \cap N\right\}}$. However, for split exact sequences the Hilbert-Samuel polynomial is always additive:

Lemma 4.9. Let $(R, \mathfrak{m})$ be a local ring and let $M, N$ be finitely generated $R$-modules. Consider the exact sequence

$$
0 \rightarrow N \rightarrow N \oplus M \rightarrow M \rightarrow 0 .
$$

Then $\chi_{N \oplus M}^{\mathfrak{q}}=\chi_{M}^{\mathfrak{q}}+\chi_{N}^{\mathfrak{q}}$ for any $\mathfrak{m}$-primary ideal $\mathfrak{q}$. 
Proof. A direct computation shows that $N \cap \mathfrak{q}^{n}(N \oplus M)=\mathfrak{q}^{n} N$ for any $n$. Using the exact sequence (4.2) the assertion follows.

Let now $D=D_{1} \cup D_{2} \subseteq \mathbb{C}^{n}$ be a not necessarily splayed divisor that is locally at a point $p=(x)$ defined by $h_{1}(x) h_{2}(x) \in \mathcal{O}=\mathbb{C}\{x\}$, with components $\left(D_{1}, p\right)=\left\{h_{1}(x)=0\right\}$ and $D_{2}=\left\{h_{2}(x)=0\right\}$. The multiplicities of $D_{i}$ at $p$ are denoted by $m_{p}\left(D_{i}\right):=\mathfrak{e}\left(\mathcal{O}_{S, p} /\left(h_{i}\right), \mathfrak{m}\right)$. The Hilbert-Samuel polynomial of $D_{i}$ at $p$ is denoted by $\chi_{D_{i}, p}:=\chi_{\mathcal{O} /\left(h_{i}\right)}^{\mathfrak{m}}$, and similarly the multiplicity and Hilbert-Samuel polynomial for $D$.

Remark 4.10. In order to compute the Hilbert-Samuel polynomial of $\mathcal{O} / I$ for any ideal $I \subseteq \mathcal{O}$, we can consider $\mathcal{O} / I$ either as a ring or as an $\mathcal{O}$-module. This does not make a difference for the Hilbert-Samuel functions, since they only depend on the graded structure of $\mathcal{O} / I$.

It is well-known that multiplicities of divisors are additive, more precisely one can show that for $D$ and $D_{i}$ defined as above, $m_{p}(D)=m_{p}\left(D_{1}\right)+m_{p}\left(D_{2}\right)$ (see e.g. [dJP00]). For splayed divisors the additivity also holds for Hilbert-Samuel polynomials:

Proposition 4.11. Let $(D, p)=\left(D_{1}, p\right) \cup\left(D_{2}, p\right)$ be splayed at $p \in S$, where $(S, p) \cong\left(\mathbb{C}^{n}, 0\right)$. Then the Hilbert-Samuel polynomials of the components $D_{1}$ and $D_{2}$ are additive, that is,

$$
\chi_{D, p}(t)+\chi_{D_{1} \cap D_{2}, p}(t)=\chi_{D_{1}, p}(t)+\chi_{D_{2}, p}(t) .
$$

Proof. Denote by $\mathcal{O}:=\mathbb{C}\{x, y\}=\mathbb{C}\left\{x_{1}, \ldots, x_{k}, y_{k+1}, \ldots, y_{n}\right\}$ the coordinate ring of $\left(\mathbb{C}^{n}, 0\right)$. There is an exact sequence (see e.g. [GP08])

$$
0 \rightarrow \mathcal{O} /\left(h_{1} h_{2}\right) \rightarrow \mathcal{O} /\left(h_{1}\right) \oplus \mathcal{O} /\left(h_{2}\right) \rightarrow \mathcal{O} /\left(h_{1}, h_{2}\right) \rightarrow 0
$$

With Facts (i) and (ii) above, the question can be reduced to leading ideals because (4.3) remains exact if we just consider the leading ideals. The divisor $D$ is splayed, so we can assume that it is defined by $g(x) h(y)$. Choosing any valid monomial ordering, one finds $L(g)=x^{\alpha}, L(h)=y^{\beta}, L((g h))=x^{\alpha} y^{\beta}$, and Fact (ii) yields $L((g, h))=\left(x^{\alpha}, y^{\beta}\right)$. From Lemma 4.9 it follows that

$$
\chi_{\mathcal{O} /\left(x^{\alpha}\right)}^{\mathfrak{m}}+\chi_{\mathcal{O} /\left(y^{\beta}\right)}^{\mathfrak{m}}=\chi_{\mathcal{O} /\left(x^{\alpha}\right) \oplus \mathcal{O} /\left(y^{\beta}\right)}^{\mathfrak{m}} .
$$

Thus it remains to prove that the Hilbert-Samuel polynomials with respect to $\mathfrak{m}$ of the exact sequence

$$
0 \rightarrow \mathcal{O} /\left(x^{\alpha} \cdot y^{\beta}\right) \rightarrow \mathcal{O} /\left(x^{\alpha}\right) \oplus \mathcal{O} /\left(y^{\beta}\right) \rightarrow \mathcal{O} /\left(x^{\alpha}, y^{\beta}\right) \rightarrow 0
$$


are additive. In order to apply the theorem of Flenner-Vogel we show that the map

$$
\operatorname{Gr}_{\mathfrak{m}}\left(\mathcal{O} /\left(x^{\alpha} y^{\beta}\right)\right) \stackrel{\varphi}{\rightarrow} \operatorname{Gr}_{\mathfrak{m}}\left(\mathcal{O} /\left(x^{\alpha}\right) \oplus \mathcal{O} /\left(y^{\beta}\right)\right)
$$

is injective. The map $\varphi$ clearly preserves the degree, so it is enough to show the assertion for a homogeneous element of degree $d$. Therefore take some $\bar{a} \in$ $\mathfrak{m}^{d}\left(\mathcal{O} /\left(x^{\alpha} y^{\beta}\right)\right) / \mathfrak{m}^{d+1}\left(\mathcal{O} /\left(x^{\alpha} y^{\beta}\right)\right)$. Consider $\bar{a}$ as an element in $\mathcal{O}$; from Grauert's division theorem it follows that $\bar{a}$ can be written as $r+\alpha_{1} x^{\alpha}+\alpha_{2} y^{\beta}$, where $r$ is the unique remainder from the division through $x^{\alpha}$ and $y^{\beta}$, and $\alpha_{1} \in \mathcal{O}$ is not divisible by $y^{\beta}$ and $\alpha_{2}$ is not divisible by $x^{\alpha}$. Suppose that $\varphi(\bar{a})=(0,0)$. Then write $\varphi(\bar{a})=\left(r+\alpha_{2} y^{\beta}, r+\alpha_{1} x^{\alpha}\right)$ in $\mathcal{O} /\left(x^{\alpha}\right) \oplus \mathcal{O} /\left(y^{\beta}\right)$. In $\mathcal{O}$ this reads as $r+\alpha_{2} y^{\beta}=c x^{\alpha}$ and $r+\alpha_{1} x^{\alpha}=c^{\prime} y^{\beta}$ for some $c, c^{\prime} \in \mathcal{O}$ with the right order. It follows that $r \in\left(x^{\alpha}, y^{\beta}\right)$. But $r$ is the unique remainder from the division through the standard basis $\left(x^{\alpha}, y^{\beta}\right)$, so $r=0$ in $\mathcal{O}$. Since $x^{\alpha}, y^{\beta}$ are clearly a regular sequence in $\mathcal{O}$, their syzygies are trivial and from the conditions on $\alpha_{1}, \alpha_{2}$ it follows that $\alpha_{1}=\alpha_{2}=0$. This implies the injectivity of $\varphi$. Hence by the theorem of Flenner-Vogel, the remainder polynomial $S$ is zero and the assertion follows.

In general the Hilbert-Samuel polynomial of a divisor $(D, p)=\left(D_{1}, p\right) \cup$ $\left(D_{2}, p\right)$ is not additive, as is seen in the following example.

Example 4.12. By Lemma 4.2.20 of [dJP00] one can explicitly compute the Hilbert-Samuel polynomial of $\mathcal{O} /(f)$, where $\mathcal{O}=\mathbb{C}\left\{x_{1}, \ldots, x_{n}\right\}$ and $\operatorname{ord}(f)=m$, namely

$$
\chi_{\mathcal{O} /(f)}^{\mathfrak{m}}(d)=\sum_{j=1}^{m}\left(\begin{array}{c}
n+d-j-1 \\
n-1
\end{array}\right) .
$$

Consider now $\left(\mathbb{C}^{2}, 0\right)$ with coordinate ring $\mathcal{O}=\mathbb{C}\{x, y\}$ and with $h_{1}=x^{2}-y$ and $h_{2}=y$. Then the germ of the divisor $(D, 0)=\left(D_{1}, 0\right) \cup\left(D_{2}, 0\right)$ that is locally given by $\left\{y\left(x^{2}-y\right)=0\right\}$ with $\left(D_{1}, 0\right)=\left\{x^{2}-y=0\right\}$ and $\left(D_{2}, 0\right)=\{y=0\}$ is not splayed. The intersection $\left(D_{1} \cap D_{2}, 0\right)$ is locally given by the ideal $\left(x^{2}, y\right)$ and coordinate ring $\mathcal{O} /\left(x^{2}, y\right)=\mathbb{C}\{x\} /\left(x^{2}\right)$. By formula (4.4) we can compute the Hilbert-Samuel polynomials of $D, D_{1}, D_{2}$ and $D_{1} \cap D_{2}$ and obtain $\chi_{D, p}(t)=2 t-1$, which is clearly not equal to $\chi_{D_{1}, p}(t)+\chi_{D_{2}, p}(t)-\chi_{D_{1} \cap D_{2}, p}(t)=t+t-2$.

One might ask if the additivity of the Hilbert-Samuel polynomials characterizes splayed divisors. However, here is a counterexample:

Example 4.13. Consider $D \subseteq \mathbb{C}^{3}$ locally defined by $g h=\left(x^{2}-y^{3}\right)\left(y^{2}-x^{2} z\right)$. Then $(D, p)$ is the union of the cylinder over a cusp $\left(D_{1}, p\right)$ and of the Whitney 
umbrella $\left(D_{2}, p\right)$. Clearly $(D, p)$ is not splayed (use for example the Leibniz property). However, $L(g)=x^{2}$ and $L(h)=y^{2}$, so the leading monomials of $g$ and $h$ are coprime and one can repeat the argument in the proof of the preceding proposition to find that

$$
\chi_{D, p}+\chi_{D_{1} \cap D_{2}, p}=\chi_{D_{1}, p}+\chi_{D_{2}, p}
$$

\section{Acknowledgments}

I thank my advisor H. Hauser for getting me interested in this topic and helping me with comments and suggestions. I thank D. Mond and L. Narváez Macarro for several discussions and comments on this work, and also P. Aluffi, M. Granger, H. Kawanoue, S. Perlega, D. B. Westra and the referee for helpful comments on an earlier version of this paper. In particular I thank O. Villamayor and his research group at the Universidad Autónoma de Madrid for their hospitality and for many discussions in which the basis for the results presented here was laid.

The author has been supported by a For Women in Science award 2011 of L'Oréal Austria, the Austrian commission for UNESCO and the Austrian Academy of Sciences and by the Austrian Science Fund (FWF) within projects J3326 and P21461. This article contains work from the author's Ph.D. dissertation at the Universität Wien.

\section{References}

[Ale86] A. G. Aleksandrov, Euler-homogeneous singularities and logarithmic differential forms, Ann. Global Anal. Geom. 4 (1986), 225-242. Z Zbl 0632.32007 MR 0910552

[Ale90] A. G. Aleksandrov, Nonisolated Saito singularities, Math. USSR Sbornik 65 (1990), 561-574. Zbl 0684.32010 MR 0981525

[AF12] P. Aluffi and E. Faber, Splayed divisors and their Chern classes, arXiv:1207.4202v2 [math.AG], 2012; J. London Math. Soc., 2013, to appear.

[Bod04] G. Bodnár, Algorithmic tests for the normal crossing property, in Automated deduction in geometry, Lecture Notes in Comput. Sci. 2930, Springer, Berlin, 2004, 1-20. Zbl 1202.14013 MR 2090399

[BEvB09] R.-O. Buchweitz, W. Ebeling, and H. C. Graf von Bothmer, Low-dimensional singularities with free divisors as discriminants. J. Algebraic Geom. 18 (2009), 371-406. Zbl 1167.14004 MR 2475818

[BM06] R.-O. Buchweitz and D. Mond, Linear free divisors and quiver representations, in Singularities and computer algebra, London Math. Soc. Lecture Note Ser. 324, Cambridge Univ. Press, Cambridge, 2006, 41-77. Zbl 1101.14013 MR 2228227

[Bud12] N. Budur, Singularity invariants related to Milnor fibers: survey, in Recent trends in zeta functions in algebra and geometry, Contemp. Math. 566, Amer. Math. Soc. 2012, 161-187. Zbl 1254.14005 MR 2858923

[CNM96] F. Castro Jiménez, L. Narváez Macarro, and D. Mond, Cohomology of the complement of a free divisor, Trans. Amer. Math. Soc. 348 (1996), 3037-3049. Zbl 0862.32021 MR 1363009 
[Dam96] J. Damon, Higher multiplicities and almost free divisors and complete intersections, Mem. Amer. Math. Soc. 123 (1996), no. 589, x+113 pp. Zbl 0867.32015 MR 1346928

[dJP00] T. de Jong and G. Pfister, Local analytic geometry, Advanced Lectures in Mathematics, Vieweg, Braunschweig, 2000. Zbl 0959.32011 MR 1760953

[Fab11] E. Faber, Normal crossings in local analytic geometry, Phd thesis, Univ. Wien, 2011.

[Fab12] E. Faber, Characterizing normal crossing hypersurfaces, arXiv:1202.6276v1 [math.AG], 2012.

[FH10] E. Faber and H. Hauser, Today's menu: geometry and resolution of singular algebraic surfaces, Bull. Amer. Math. Soc. 47 (2010), 373-417. Zbl 1235.14016 MR 2651084

[FV93] H. Flenner and W. Vogel, On multiplicities of local rings, Manuscripta Math. 78 (1993), 85-97. Zbl 0794.13016 MR 1201763

[GH85] T. Gaffney and H. Hauser, Characterizing singularities of varieties and of mappings, Invent. Math. 81 (1985), 427-447. Zbl 0627.14004 MR 0807067

[GP08] G.-M. Greuel and G. Pfister, A singular introduction to commutative algebra, Springer, Berlin, 2008. Zbl 1133.13001 MR 2363237

[GS09] M. Granger and M. Schulze, Initial logarithmic Lie algebras of hypersurface singularities, J. Lie Theory 19 (2009), 209-221. Zbl 1190.32023 MR 2572124

[HM93] H. Hauser and G. Müller, Affine varieties and Lie algebras of vector fields, Manuscripta Math. 80 (1993), 309-337. Zbl 0805.14004 MR 1240653

[Li09] L. Li, Wonderful compactification of an arrangement of subvarieties, Michigan Math. J. 58 (2009), 535-563. Zbl 1187.14060 MR 2595553

[MY82] J. N. Mather and S. S.-T. Yau, Classification of isolated hypersurface singularities by their moduli algebras, Invent. Math. 69 (1982), 243-251. Zbl 0499.32008 MR 0674404

[Mat86] H. Matsumura, Commutative ring theory, Cambridge Stud. Adv. Math. 8, Cambridge Univ. Press, Cambridge, 1986. Zbl 0603.13001 MR 0879273

[MS10] D. Mond and M. Schulze, Adjoint divisors and free divisors, arXiv:1001.1095v2 [math.AG], 2010.

[OT92] P. Orlik and H. Terao, Arrangements of hyperplanes, Grundlehren Math. Wiss. 300, Springer, Berlin, 1992. Zbl 0757.55001 MR 1217488

[Sai80] K. Saito, Theory of logarithmic differential forms and logarithmic vector fields, J. Fac. Sci. Univ. Tokyo 27 (1980), 265-291. Zbl 0496.32007 MR 0586450

[Sai81] K. Saito, Primitive forms for a universal unfolding of a function with an isolated critical point, J. Fac. Sci. Univ. Tokyo Sect. IA Math. 28 (1981), 775-792. Zbl 0523.32015 MR 0656053

[Sek08] J. Sekiguchi, Three dimensional Saito free divisors and singular curves, J. Sib. Fed. Univ. Math. Phys. 1 (2008), 33-41.

[Sim06] A. Simis, Differential idealizers and algebraic free divisors, in Commutative algebra, Lecture Notes in Pure Appl. Math. 244, Dekker, 2006, 211-226. Zbl 1099.13030 MR 2184799 